\title{
On numerical solution to fractional non-linear oscillatory equations
}

\author{
Mehmet Merdan • Ahmet Gökdoğan • \\ Ahmet Yildirim
}

Received: 15 May 2011 / Accepted: 31 October 2012 / Published online: 28 November 2012

(C) The Author(s) 2012. This article is published with open access at Springerlink.com

\begin{abstract}
In this article, the multi-step differential transform method (MsDTM) is applied to give approximate solutions of nonlinear ordinary differential equation such as fractional-non-linear oscillatory and vibration equations. The results indicate that the method is very effective and sufficient for solving nonlinear differential equations of fractional order.
\end{abstract}

Keywords Multi-step differential transform method · Rayleigh · Duffing · Vander Pol equation and vibration $\cdot$ Non-linear oscillatory equations

\section{Introduction}

The Rayleigh equation determines a typical non-linear system with one degree of freedom which admits

M. Merdan ( $\varangle)$. A. Gökdoğan

Department of Mathematical Engineering, Gümüşhane

University, 29100 Gümüşhane, Turkey

e-mail:mmerdan@gumushane.edu.tr

A. Gökdoğan
e-mail: gokdogan@gumushane.edu.tr

A. Yildirim

Department of Mathematics, Ege University, 35000, İzmir, Turkey

e-mail: ahmet.yildirim@ege.edu.tr

A. Yildirim

Department of Mathematics and Statistics, University of South Florida, Tampa, FL 33620-5700, USA auto-oscillations. This equation was named after Lord Rayleigh who investigated equations of this type related to problems in acoustics [1]. The special case of the Rayleigh equation for is the Vander Pol equation [2]. In general, the Duffing equation [3] does not admit an exact symbolic solution. However, it is resolved many approximate methods $[4,5]$.

The differential transform method (DTM) is a numerical as well as analytical method for solving integral equations, ordinary, partial differential equations and differential equation systems. The method provides the solution in terms of convergent series with easily computable components. The concept of the differential transform was first proposed by Zhou [6] and its main application concern with both linear and nonlinear initial value problems in electrical circuit analysis. The DTM gives exact values of the nth derivative of an analytic function at a point in terms of known and unknown boundary conditions in a fast manner. This method constructs, for differential equations, an analytical solution in the form of a polynomial. It is different from the traditional high order Taylor series method, which requires symbolic computations of the necessary derivatives of the data functions. The Taylor series method is computationally taken long time for large orders. The DTM is an iterative procedure for obtaining analytic Taylor series solutions of differential equations. Different applications of DTM can be found in [7-29].

However, DTM has some drawbacks. By using the DTM, we obtain a series solution, actually a truncated 
series solution. This series solution does not exhibit the real behaviors of the problem but gives a good approximation to the true solution in a very small region. To overcome the shortcoming, MsDTM was presented in [30, 31]. On the other hand, MsDTM has also some drawbacks. By using the DTM, the interval $[0, T]$ is divided into $M$ sub-interval and the series solutions is obtained in $t \in\left[t_{i}, t_{i+1}\right], i=0,1, \ldots, M-1$. In some problems, interval $[0, T]$ can be required a very small sub-division of intervals. In this case, both the solution time lengthens and series solutions are obtained for a great number of sub-intervals.

The main aim of this paper is to extend the application of the multi-step differential transform method $[30,31]$ to solve a fractional order non-linear oscillator and vibration equation.

This paper is organized as follows:

In Sects. 2 and 3, we describe fractional DTM and multi-step DTM briefly. To show in efficiency of this method, we give some examples and numerical results in Sect. 4. The conclusions are then given in the final Sect. 5.

\section{Fractional differential transform method}

Consider a general system of fractional differential equations:

$$
D^{m} x(t)+f(x) D_{*}^{\alpha} x(t)+h\left(t, x, x^{\prime}, x^{\prime \prime}, \ldots\right)=0,
$$

where $D_{*}^{\alpha}$ is the derivative of $x$ of order $\alpha$ in the sense of Caputo and $m-1<\alpha \leq m$, subject to the initial conditions

$x^{(i)}(0)=c_{i}, \quad i=0,1, \ldots, m-1$.

In this paper, we introduce the multi-step fractional differential transform method used in this paper to obtain approximate analytical solutions for the fractional differential equations (1). This method has been developed in [32] as follows:

$$
\begin{aligned}
D_{x_{0}}^{q} f(x)= & \frac{1}{\Gamma(m-q)} \frac{d^{m}}{d x^{m}} \\
& \times\left[\int_{x_{0}}^{x} \frac{f(t)}{(x-t)^{1+q-m}} d t\right],
\end{aligned}
$$

for $m-1 \leq q<m, m \in Z^{+}, x>x_{0}$. Let us expand the analytical and continuous function $f(x)$ in terms of fractional power series as follows: $f(x)=\sum_{k=0}^{\infty} F(k)\left(x-x_{0}\right)^{\frac{k}{\alpha}}$,

where $\alpha$ is the order of fraction and $F(k)$ is the fractional differential transform of $f(x)$.

In order to avoid fractional initial and boundary conditions, we define the fractional derivative in the Caputo sense. The relation between the RiemannLiouville operator and Caputo operator is given by

$$
\begin{aligned}
& D_{* x_{0}}^{q} f(x) \\
& \quad=D_{x_{0}}^{q}\left[f(x)-\sum_{k=0}^{m-1} \frac{1}{k !}\left(x-x_{0}\right)^{k} f^{(k)}\left(x_{0}\right)\right] .
\end{aligned}
$$

Setting $f(x)-\sum_{k=0}^{m-1} \frac{1}{k !}\left(x-x_{0}\right)^{k} f^{(k)}\left(x_{0}\right)$ in Eq. (3) and using Eq. (5), we obtain fractional derivative in the Caputo sense [32-34] as follows:

$$
\begin{array}{rl}
D_{* x_{0}}^{q} & f(x) \\
= & \frac{1}{\Gamma(m-q)} \frac{d^{m}}{d x^{m}} \\
& \times\left[\int_{x_{0}}^{x}\left\{\frac{f(t)-\sum_{k=0}^{m-1} \frac{1}{k !}\left(t-x_{0}\right)^{k} f^{(k)}\left(x_{0}\right)}{(x-t)^{1+q-m}}\right\} d t\right]
\end{array}
$$

since the initial conditions are implemented are implemented to the integer order derivatives, the transform of the initial conditions are defined as follows:

$$
\begin{gathered}
F(k)= \begin{cases}\text { if } \frac{k}{\alpha} \in Z^{+}, & \frac{1}{\left(\frac{k}{\alpha}\right) !}\left[\frac{d^{\frac{k}{\alpha}} f(x)}{d x^{\frac{k}{\alpha}}}\right]_{x=x_{0}} \\
\text { if } \frac{k}{\alpha} \notin Z^{+} & 0,\end{cases} \\
\text { for } k=0,1,2, \ldots,(q \alpha-1)
\end{gathered}
$$

where, $q$ is the order of fractional differential equation considered. The following theorems that can be deduced from Eqs. (4) and (5) are given below, for proofs and detailed see [34-36].

Theorem 1 If $z(t)=x(t) \pm y(t)$, then $Z(k)=X(k) \pm$ $Y(k)$.

Theorem 2 If $z(t)=c y(t)$, then $Z(k)=c Y(k)$.

Theorem 3 If $z(t)=x(t) y(t)$ and $u(t)=f(t) g(t) h(t)$, then

$Z(k)=\sum_{k_{1}=0}^{k} X\left(k_{1}\right) Y\left(k-k_{1}\right)$ 
and

$U(k)=\sum_{k_{2}=0}^{k} \sum_{k_{1}=0}^{k_{2}} F\left(k_{1}\right) G\left(k_{2}-k_{1}\right) H\left(k-k_{2}\right)$.

Theorem 4 If $z(t)=\left(t-t_{0}\right)^{n}$, then $Z(k)=\delta(k-\alpha p)$ where

$\delta(k)= \begin{cases}1 & k=0 \\ 0 & k \neq 0\end{cases}$

Theorem 5 If $z(t)=D_{t_{0}}^{q}[g(t)]$ then $Z(k)=$ $\frac{\Gamma\left(q+1+\frac{k}{\alpha}\right)}{\Gamma\left(1+\frac{k}{\alpha}\right)} G(k+\alpha q)$.

According to fractional DTM, by taking differential transformed both sides of the equations given Eqs. (1) and (2) is transformed as follows:

$$
\begin{aligned}
& \frac{\Gamma\left(\frac{k+q+m q}{q}\right)}{\Gamma\left(\frac{k+q}{q}\right)} X(k+m q) \\
& \quad+F(r) \frac{\Gamma\left(\frac{k-r+q+p}{q}\right)}{\Gamma\left(\frac{k-r+q}{q}\right)} X(k-r+p)+H(k)=0,
\end{aligned}
$$

$X(q i)=c_{i}, \quad i=0,1, \ldots, m-1$

where, $\alpha=\frac{p}{q}$ is the order of fractional differential equation considered. Therefore, according to DTM the $N$-term approximations for (1) can be expressed as

$x(t)=\sum_{k=1}^{N} X(k) t^{\alpha k}$.

\section{Solutions by MsDTM}

Let $[0, T]$ be the interval over which we want to find the solution of the initial value problem (1). In actual applications of the DTM, the approximate solution of the initial value problem (1)-(2) can be expressed by the finite series,

$x(t)=\sum_{i=0}^{N} b_{i} t^{\alpha i}, \quad t \in[0, T]$.

Assume that the interval $[0, T]$ is divided into $N$ subintervals $\left[t_{n-1}, t_{n}\right], n=1,2, \ldots, N$ of equal step size $h=T / N$ by using the nodes $t_{n}=n h$. The main ideas of the multi-step DTM are as follows [30, 31]. First, we apply the DTM to Eq. (1) over the interval $\left[0, t_{1}\right]$, we will obtain the following approximate solution,

$x_{1}(t)=\sum_{i=0}^{N} b_{1 i} t^{\alpha i}, \quad t \in\left[0, t_{1}\right]$

using the initial conditions $x_{1}^{(k)}(0)=d_{k}$. For $n \geq 2$ and at each subinterval $\left[t_{n-1}, t_{n}\right]$ we will use the initial conditions $x_{n}^{(k)}\left(t_{n-1}\right)=x\left(t_{n-1}\right)$ and apply the DTM to Eq. (1) over the interval $\left[t_{n-1}, t_{n}\right]$, where $t_{i}$ in Eq. (12) is replaced by $t_{n-1}$. The process is repeated and generates a sequence of approximate solutions $x_{n}(t), n=$ $1,2, \ldots, N$ for the solution $x(t)$,

$x_{n}(t)=\sum_{i=0}^{N} b_{n i}\left(t-t_{n-1}\right)^{\alpha i}, \quad t \in\left[t_{n}, t_{n+1}\right]$

In fact, the multi-step DTM assumes the following solution,

$x(t)= \begin{cases}x_{1}(t), & t \in\left[0, t_{1}\right], \\ x_{2}(t), & t \in\left[t_{1}, t_{2}\right], \\ \vdots & \\ x_{N}(t), & t \in\left[t_{N-1}, t_{N}\right] .\end{cases}$

\section{Applications}

Example 1 (The Duffing equation) The Duffing equation is a non-linear second-order differential equation as follows:

$\frac{d^{\alpha} x}{d t^{\alpha}}+x+\varepsilon x^{3}=0$

$x(0)=0, \quad x^{\prime}(0)=1$.

We will apply classic DTM and the multi-step DTM to nonlinear ordinary differential equation (15). Applying classic DTM for Eq. (15)

$$
\begin{aligned}
& \frac{\Gamma\left(\frac{k+q+p}{q}\right)}{\Gamma\left(\frac{k+q}{q}\right)} X(k+p)+X(k) \\
& \quad+\varepsilon \sum_{k_{2}=0}^{k} \sum_{k_{1}=0}^{k_{2}} X\left(k_{1}\right) X\left(k_{2}-k_{1}\right) X\left(k-k_{2}\right)=0, \\
& X(0)=0, \quad X(q)=1,
\end{aligned}
$$

where $X_{i}(n)$, for $n=1, \ldots, M$, satisfy the following recurrence relations, 

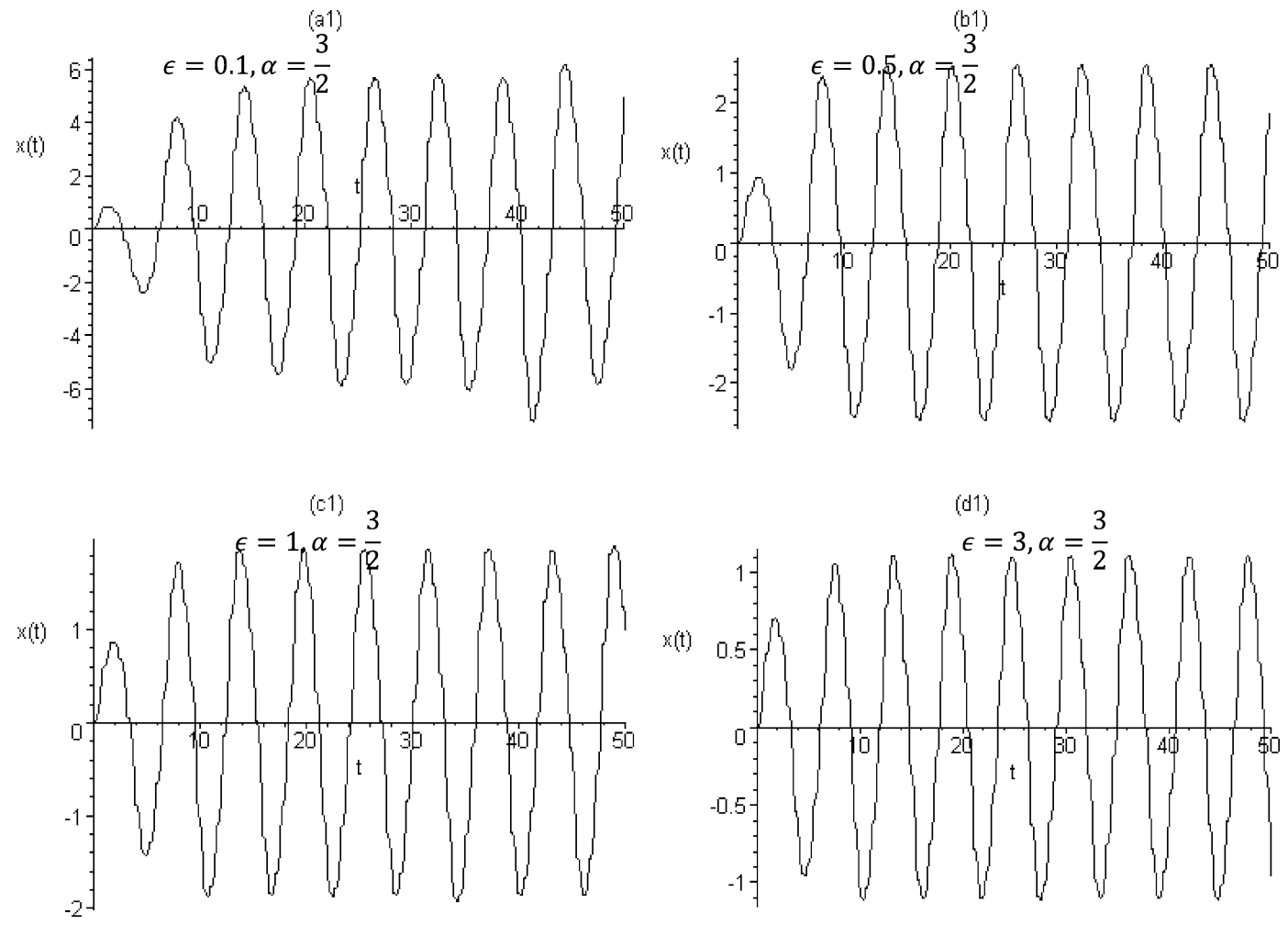

Fig. 1 Plots of displacement $x$ versus time $t$ for $\alpha=\frac{3}{2}$ and different value of $\varepsilon$

$$
\begin{aligned}
& \frac{\Gamma\left(\frac{k+q+p}{q}\right)}{\Gamma\left(\frac{k+q}{q}\right)} X_{i}(k+p)+X_{i}(k) \\
& \quad+\varepsilon \sum_{k_{2}=0}^{k} \sum_{k_{1}=0}^{k_{2}} X_{i}\left(k_{1}\right) X_{i}\left(k_{2}-k_{1}\right) X_{i}\left(k-k_{2}\right)=0, \\
& X_{0}(0)=0, \quad X_{0}(q)=1 \\
& X_{i+1}(0)=X_{i}\left(t^{*}\right), \quad X_{i+1}(q)=X_{i}\left(t^{*}\right) \\
& t \in\left[t_{i}, t_{i+1}\right], \quad t^{*}=t_{i}, \quad i=0, \ldots, M-1
\end{aligned}
$$

By applying the multi-step DTM to Eq. (15) is obtained Eq. (18) as following:

$x(t)= \begin{cases}\sum_{k=0}^{N} X_{0}(k) t^{k \alpha}, & t \in\left[t_{0}, t_{1}\right] \\ \sum_{k=0}^{N} X_{1}(k) t^{k \alpha}, & t \in\left[t_{1}, t_{2}\right] \\ \vdots & \\ \sum_{k=0}^{N} X_{M}(k) t^{k \alpha}, & t \in\left[t_{M-1}, t_{M}\right]\end{cases}$

The solutions of Eq. (15) corresponding to $\varepsilon=$ $0.1,0.5,1,3$ and $\alpha=\frac{3}{2}$, respectively, are shown in Fig. 1. The results show that in the interval $0<\varepsilon \leq 3$ the frequency increases with increases $\varepsilon$.
The solutions of Eq. (15) corresponding to $\varepsilon=$ $0.1,0.5,1,3$ and $\alpha=\frac{5}{3}$, respectively, are shown in Fig. 2. The results show that in the interval $0<\varepsilon \leq 3$ the frequency increases with increases $\varepsilon$. The results obtained from in Figs. 1 and 2 show that in the interval $1<\alpha<2$ the frequency decreases with increases.

Table 1 shows the approximate solutions for Eq. (15) obtained for different values of a using MsDTM with the Runge Kutta method. From the numerical results in Table 1, it is clear that the approximate solutions are in high agreement with the RKM solutions, when $\alpha=2$.

Example 2 (The Vander Pol equation) Consider the following Vander Pol equation.

$$
\begin{aligned}
& \frac{d^{2} x}{d t^{2}}+\mu\left(x^{2}-1\right) \frac{d^{\alpha} x}{d t^{\alpha}}+x=a \sin (w t), \\
& y(0)=0, \quad y^{\prime}(0)=0 .
\end{aligned}
$$


(a1)

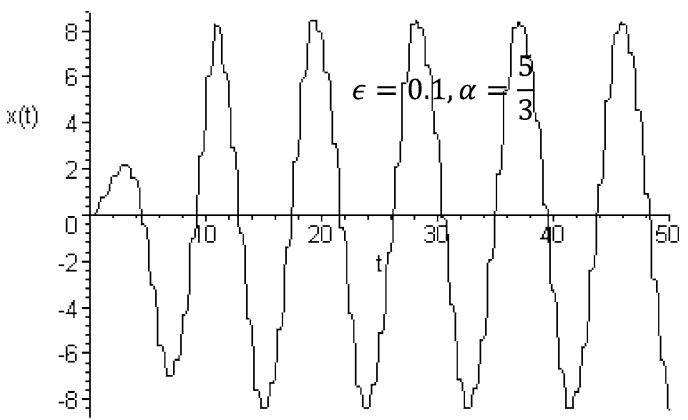

(c1)

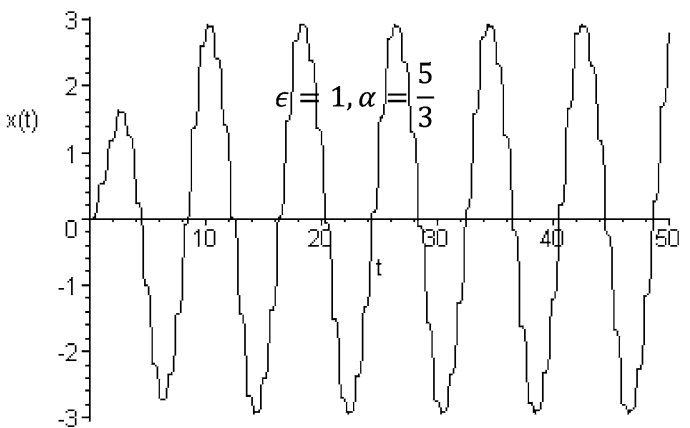

(b1)

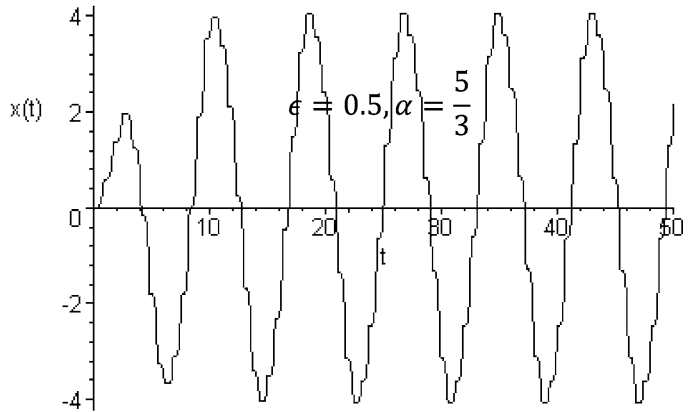

(d1)

Fig. 2 Plots of displacement $x$ versus time $t$ for $\alpha=\frac{5}{3}$ and different value of $\varepsilon$

Table 1 Approximate solutions for Duffing equation (15), when $\varepsilon=0.1$, obtained using multi-step DTM and RK4 method

\begin{tabular}{|c|c|c|c|c|}
\hline \multirow[t]{2}{*}{$t$} & \multicolumn{2}{|l|}{ Multi-step DTM } & \multicolumn{2}{|l|}{ RK4 } \\
\hline & $x(t)_{\alpha=2}$ & $d x / d t_{\alpha=2}$ & $x(t)_{\alpha=2}$ & $d x / d t_{\alpha=2}$ \\
\hline 0 & 0 & 1 & 0 & 1 \\
\hline 1 & 0.8376372563 & 0.5232020151 & 0.8376388747 & 0.5232076636 \\
\hline 2 & 0.8554467235 & -0.4913510782 & 0.8554611051 & -0.4913336268 \\
\hline 3 & 0.0350962937 & -0.9993840456 & 0.0351219580 & -0.9993829956 \\
\hline 4 & -0.8187234745 & -0.5542727657 & -0.8187105403 & -0.5543002990 \\
\hline 5 & -0.8721250801 & 0.4587619764 & -0.8721505150 & 0.4587203097 \\
\hline 6 & -0.0701493098 & 0.9975361877 & -0.0702005699 & 0.9975322880 \\
\hline 7 & 0.7987332983 & 0.5845233024 & 0.7987042607 & 0.5845715230 \\
\hline 8 & 0.8876471393 & -0.4254787133 & 0.8876819044 & -0.4254118927 \\
\hline 9 & 0.1051158328 & -0.9944573509 & 0.1051925110 & -0.9944487987 \\
\hline 10 & -0.7776961948 & -0.6139158410 & -0.7776495628 & -0.6139834720 \\
\hline
\end{tabular}


(a1)

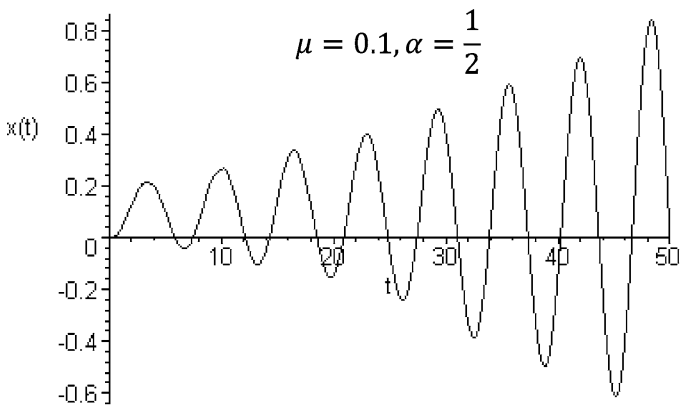

(c1)

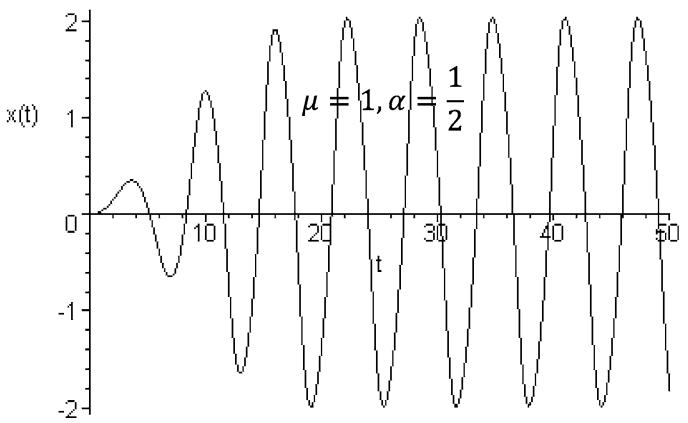

(b1)

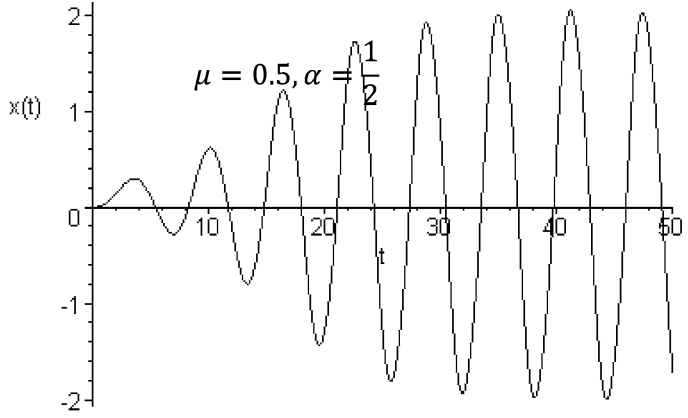

(d1)

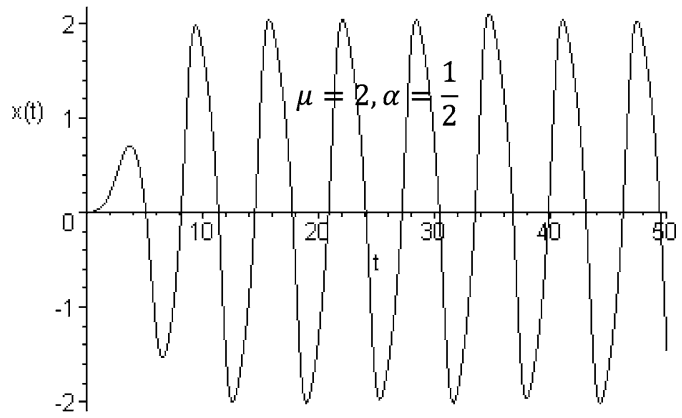

Fig. 3 Plots of displacement $x$ versus time $t$ for $\alpha=\frac{1}{2}$ and different value of $\mu$

Taking classic-DTM of both sides Eq. (19), we obtain the following recurrence relation:

$$
\begin{aligned}
& \frac{\Gamma\left(\frac{k+q+r q}{q}\right)}{\Gamma\left(\frac{k+q}{q}\right)} X(k+r q)-\mu \frac{\Gamma\left(\frac{k+q+p}{q}\right)}{\Gamma\left(\frac{k+q}{q}\right)} X(k+p) \\
& \quad+\mu \sum_{k_{2}=0}^{k} \sum_{k_{1}=0}^{k_{2}} \frac{\Gamma\left(\frac{k-k_{2}+q+p}{q}\right)}{\Gamma\left(\frac{k-k_{2}+q}{q}\right)} \\
& \quad \times X\left(k_{1}\right) X\left(k_{2}-k_{1}\right) X\left(k-k_{2}+p\right)+X(k) \\
& =\frac{a \sum_{i=0}^{\infty}(-1)^{i} w^{2 i+1} \delta(k-(2 i+1) q)}{(2 i+1) !} \\
& X(0)=0, \quad X(q)=0
\end{aligned}
$$

where $A(k)$ is the fractional differential transform of $\sin (w t)$ that can be obtained using Eq. (4) [34] as

$A(K)=\sum_{i=0}^{\infty} \frac{(-1)^{i} w^{2 i+1} \delta(k-(2 i+1) q)}{(2 i+1) !}$,

According to the multi-step DTM, the series solution for Vander Pol equation (19) is given by,

$$
x(t)= \begin{cases}\sum_{k=0}^{N} X_{0}(k) t^{k \alpha}, & t \in\left[t_{0}, t_{1}\right] \\ \sum_{k=0}^{N} X_{1}(k) t^{k \alpha}, & t \in\left[t_{1}, t_{2}\right] \\ \vdots & \\ \sum_{k=0}^{N} X_{M}(k) t^{k \alpha}, & t \in\left[t_{M-1}, t_{M}\right]\end{cases}
$$

where $X_{i}(n)$, for $n=1, \ldots, M$, satisfy the following recurrence relations,

$$
\begin{aligned}
& \frac{\Gamma\left(\frac{k+q+r q}{q}\right)}{\Gamma\left(\frac{k+q}{q}\right)} X_{i}(k+r q)-\mu \frac{\Gamma\left(\frac{k+q+p}{q}\right)}{\Gamma\left(\frac{k+q}{q}\right)} X_{i}(k+p) \\
& \quad+\mu \sum_{k_{2}=0}^{k} \sum_{k_{1}=0}^{k_{2}} \frac{\Gamma\left(\frac{k-k_{2}+q+p}{q}\right)}{\Gamma\left(\frac{k-k_{2}+q}{q}\right)} X_{i}\left(k_{1}\right) X_{i}\left(k_{2}-k_{1}\right) \\
& \quad \times X_{i}\left(k-k_{2}+p\right)+X_{i}(k) \\
& =\frac{a \sum_{i=0}^{\infty}(-1)^{i} w^{2 i+1} \delta(k-(2 i+1) q)}{(2 i+1) !} \\
& X_{0}(0)=0, \quad X_{0}(q)=0 \\
& X_{i+1}(0)=X_{i}\left(t^{*}\right), \quad X_{i+1}(q)=X_{i}\left(t^{*}\right) \\
& t \in\left[t_{i}, t_{i+1}\right], \quad t^{*}=t_{i}, \quad i=0, \ldots, M-1
\end{aligned}
$$

Figure 3 shows the approximate solutions for $\alpha=$ $\frac{1}{2}, a=1.31, w=0.5$ and $\mu=0.1,0.5,1,2$. The so- 


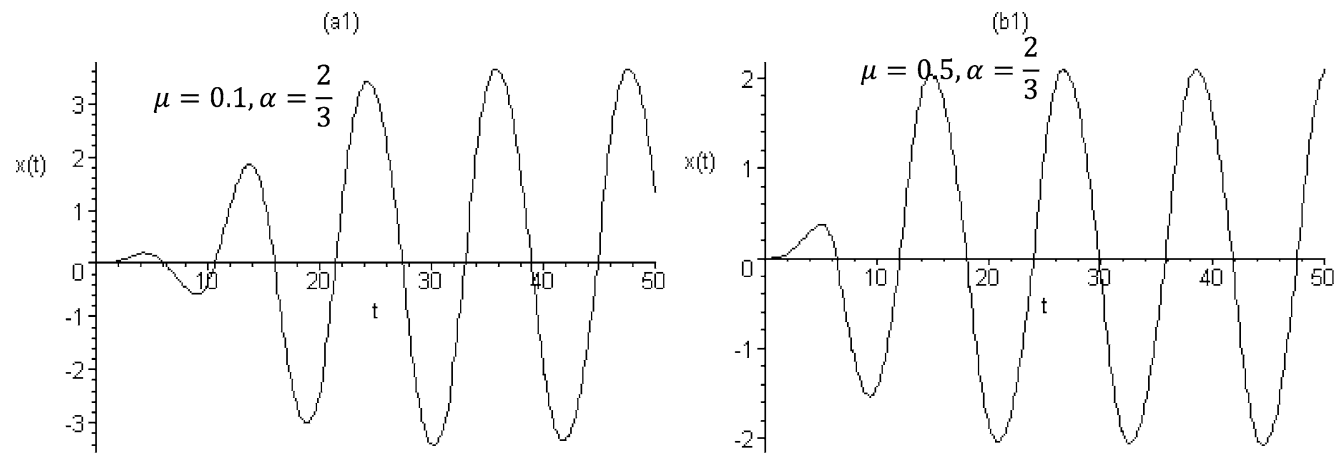

(c1)

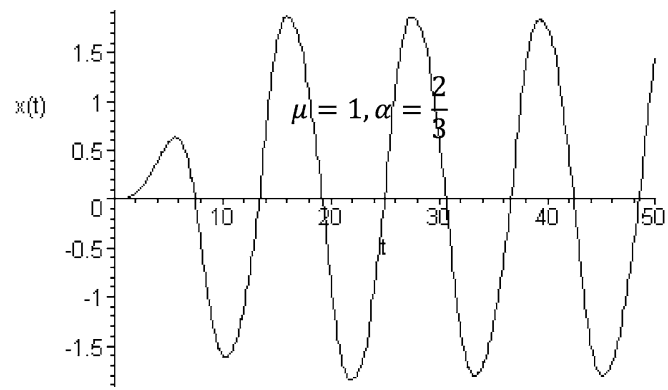

(d1)

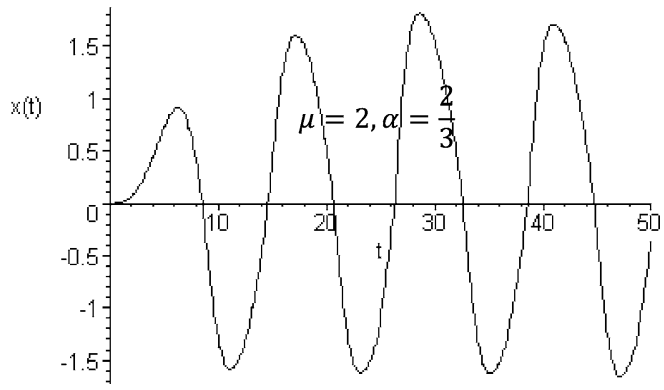

Fig. 4 Plots of displacement $x$ versus time $t$ for $\alpha=\frac{2}{3}$ and different value of $\mu$

Table 2 Approximate solutions for Vander Pol equation (19), when $\mu=0.1$, obtained using multi-step DTM and RK4 method

\begin{tabular}{|c|c|c|c|c|}
\hline \multirow[t]{2}{*}{$t$} & \multicolumn{2}{|l|}{ Multi-step DTM } & \multicolumn{2}{|l|}{ RK4 } \\
\hline & $x(t)_{\alpha=1}$ & $d x / d t_{\alpha=1}$ & $x(t)_{\alpha=1}$ & $d x / d t_{\alpha=1}$ \\
\hline 0 & 0 & 2 & 0 & 2 \\
\hline 1 & 1.719751383 & 1.063295856 & 1.719749695 & 1.0633132977 \\
\hline 2 & 1.783892507 & -0.865320790 & 1.783923664 & -0.8652903894 \\
\hline 3 & 0.284103929 & -1.952361546 & 0.284154303 & -1.9523496436 \\
\hline 4 & -1.548253041 & -1.327237126 & -1.548211594 & -1.3272875319 \\
\hline 5 & -1.891558725 & 0.626883710 & -1.891597949 & 0.6268028836 \\
\hline 6 & -0.558832003 & 1.869650073 & -0.558928085 & 1.8696156737 \\
\hline 7 & 1.341006145 & 1.557825117 & 1.340916063 & 1.5578950364 \\
\hline 8 & 1.963551148 & -0.369392450 & 1.963585131 & -0.3692566155 \\
\hline 9 & 0.819518662 & -1.757242588 & 0.819653884 & -1.7571769092 \\
\hline 10 & -1.103543561 & -1.745624504 & -1.103398523 & -1.7456984223 \\
\hline
\end{tabular}

lutions of Eq. (19) corresponding to $\mu=0.1,0.5,1,2$ and $\alpha=\frac{2}{3}$, respectively, are shown in Fig. 4. The results indicate that in the interval $0<\mu \leq 2$ the frequency increases with increases $\mu$. The results obtained from in Figs. 3 and 4 show that in the in- terval $0<\alpha<1$ the frequency decreases with increases.

Table 2 shows the approximate solutions for Eq. (19) obtained for different values of a using MsDTM with the Runge Kutta Method. From the numer- 
ical results in Table 2, it is clear that the approximate solutions are in high agreement with the RKM solutions, when $\alpha=1$.

Example 3 (Fractional Rayleigh differential equation) Consider the following fractional Rayleigh differential equation.

$\frac{d^{2} x}{d t^{2}}=-x-\varphi\left(1-\frac{1}{3}\left(\frac{d^{\alpha} x}{d t^{\alpha}}\right)^{2}\right) \frac{d x}{d t}$,

$x(0)=1, \quad x^{\prime}(0)=0$.

Taking classic-DTM of both sides Eq. (23), we obtain the following recurrence relation:

$$
\begin{aligned}
& \frac{\Gamma\left(\frac{k+q+r q}{q}\right)}{\Gamma\left(\frac{k+q}{q}\right)} X(k+r q) \\
& \quad=-X(k)-\varphi(k+1) X(k+1) \\
& \quad+\frac{\varphi}{3} \sum_{k_{2}=0}^{k} \sum_{k_{1}=0}^{k_{2}} \frac{\Gamma\left(\frac{k_{2}-k_{1}+q+p}{q}\right)}{\Gamma\left(\frac{k_{2}-k_{1}+q}{q}\right)} \frac{\Gamma\left(\frac{k-k_{2}+q+p}{q}\right)}{\Gamma\left(\frac{k-k_{2}+q}{q}\right)} \\
& \quad \times X\left(k_{1}\right) X\left(k_{2}-k_{1}+p\right) X\left(k-k_{2}+p\right), \\
& X(0)=1, \quad X(q)=0 .
\end{aligned}
$$

According to the multi-step DTM, the series solution for Rayleigh equation (23) is given by,

$$
x(t)= \begin{cases}\sum_{k=0}^{N} X_{0}(k) t^{k \alpha}, & t \in\left[t_{0}, t_{1}\right] \\ \sum_{k=0}^{N} X_{1}(k) t^{k \alpha}, & t \in\left[t_{1}, t_{2}\right] \\ \vdots & \\ \sum_{k=0}^{N} X_{M}(k) t^{k \alpha}, & t \in\left[t_{M-1}, t_{M}\right]\end{cases}
$$

where $X_{i}(n)$, for $n=1, \ldots, M$, satisfy the following recurrence relations,

$$
\begin{aligned}
& \frac{\Gamma\left(\frac{k+q+r q}{q}\right)}{\Gamma\left(\frac{k+q}{q}\right)} X_{i}(k+r q) \\
& \quad=-X_{i}(k)-\varphi(k+1) X_{i}(k+1) \\
& \quad+\frac{\varphi}{3} \sum_{k_{2}=0}^{k} \sum_{k_{1}=0}^{k_{2}} \frac{\Gamma\left(\frac{k_{2}-k_{1}+q+p}{q}\right)}{\Gamma\left(\frac{k_{2}-k_{1}+q}{q}\right)} \frac{\Gamma\left(\frac{k-k_{2}+q+p}{q}\right)}{\Gamma\left(\frac{k-k_{2}+q}{q}\right)} \\
& \quad \times X_{i}\left(k_{1}\right) X_{i}\left(k_{2}-k_{1}+p\right) X_{i}\left(k-k_{2}+p\right) \\
& X_{0}(0)=1, \quad X_{0}(q)=1 \\
& X_{i+1}(0)=X_{i}\left(t^{*}\right), \quad X_{i+1}(q)=X_{i}\left(t^{*}\right) \\
& t \in\left[t_{i}, t_{i+1}\right], \quad t^{*}=t_{i}, \quad i=0, \ldots, M-1
\end{aligned}
$$

Figure 5 shows the approximate solutions for $\alpha=\frac{1}{2}$ and $\varphi=0.1,0.5,1,2$. The solutions of Eq. (23) corresponding to $\varphi=0.1,0.5,1,2$ and $\alpha=\frac{1}{4}$, respectively, are shown in Fig. 6. The results indicate that in the interval $0<\varphi \leq 2$ the frequency decreases with increases $\varphi$. The results obtained from in Figs. 5 and 6 show that in the interval $0<\alpha<1$ the frequency decreases with increases.

Table 3 shows the approximate solutions for Eq. (23) obtained for different values of a using MsDTM with the Runge Kutta Method. From the numerical results in Table 3, it is clear that the approximate solutions are in high agreement with the RKM solutions, when $\alpha=1$.

Example 4 (Vibration differential equation) Consider the following vibration equation with fractional damping, with one degree of freedom [37]:

$D^{2} x(t)+\frac{c}{m} D^{\alpha} x(t)+\frac{k}{m} x(t)=f_{0} \sin \left(w_{e} t\right)$,

$x(0)=\frac{1}{4}, \quad x^{\prime}(0)=0$,

where $D=\frac{d}{d t}$ is the differential operator. Another common form of Eq. (27) is

$D^{2} x(t)+2 \mu w_{n}^{2-\alpha} D^{\alpha} x(t)+w_{n}^{2} x(t)=f_{0} \sin \left(w_{e} t\right)$,

with

$2 \mu w_{n}^{2-\alpha}=\frac{c}{m}, \quad \alpha=\frac{p}{q}$,

$r=2 \quad$ and $\quad w_{n}^{2}=\frac{k}{m}$.

Taking classic-DTM of both sides Eq. (28), we obtain the following recurrence relation:

$$
\begin{aligned}
& \frac{\Gamma\left(\frac{k+q+r q}{q}\right)}{\Gamma\left(\frac{k+q}{q}\right)} X(k+r q) \\
& \quad+2 \mu w_{n}^{2-\frac{p}{q}} \frac{\Gamma\left(\frac{k+q+p}{q}\right)}{\Gamma\left(\frac{k+q}{q}\right)} X(k+p)+w_{n}^{2} X(k) \\
& =\frac{f_{0} \sum_{i=0}^{\infty}(-1)^{i} w_{e}^{2 i+1} \delta(k-(2 i+1) q)}{(2 i+1) !} \\
& X(0)=\frac{1}{4}, \quad X(q)=0 .
\end{aligned}
$$

According to the multi-step DTM, the series solution for vibration equation (28) is given by, 

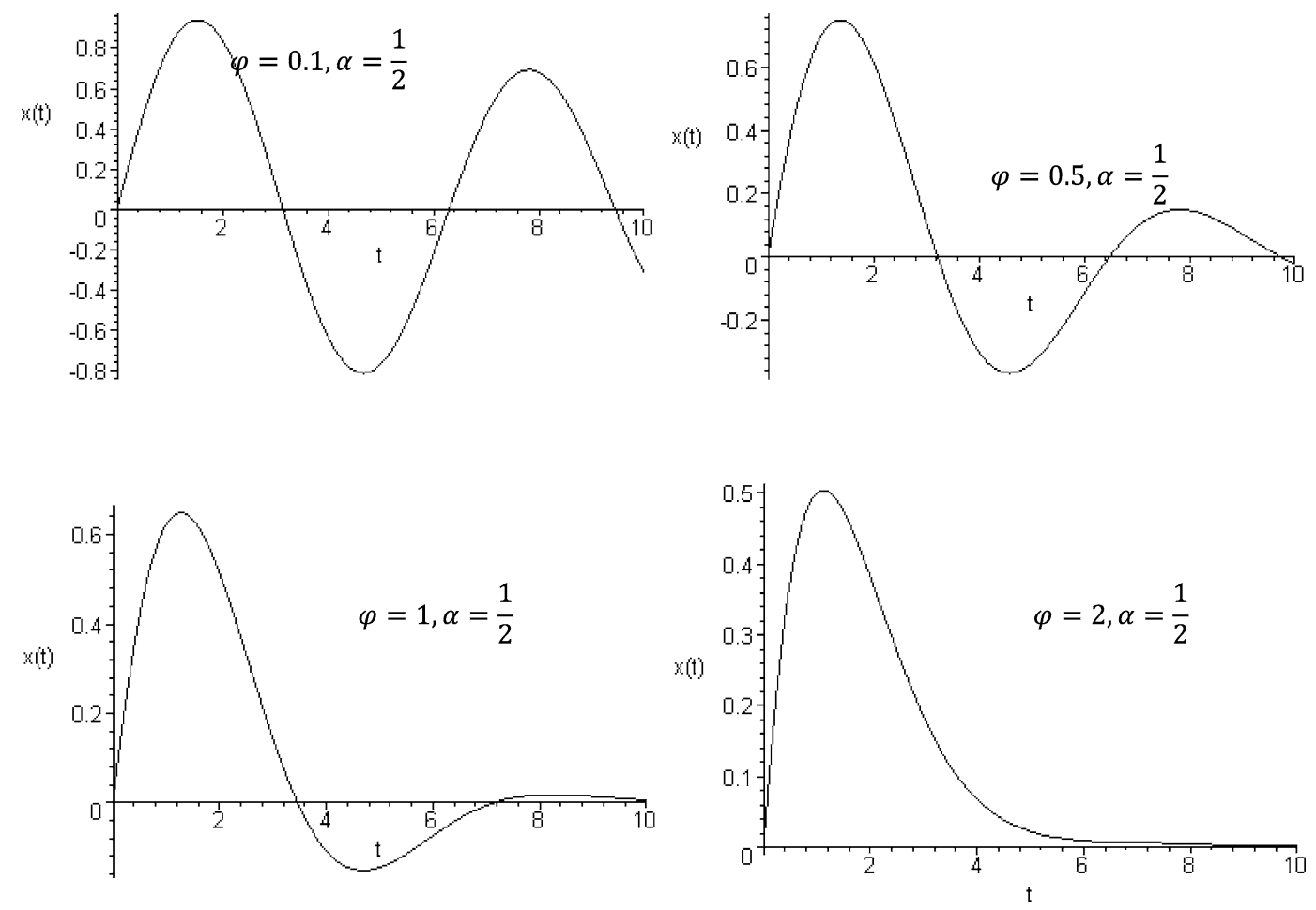

Fig. 5 Plots of displacement $x$ versus time $t$ for $\alpha=\frac{1}{2}$ and different value of $\varphi$
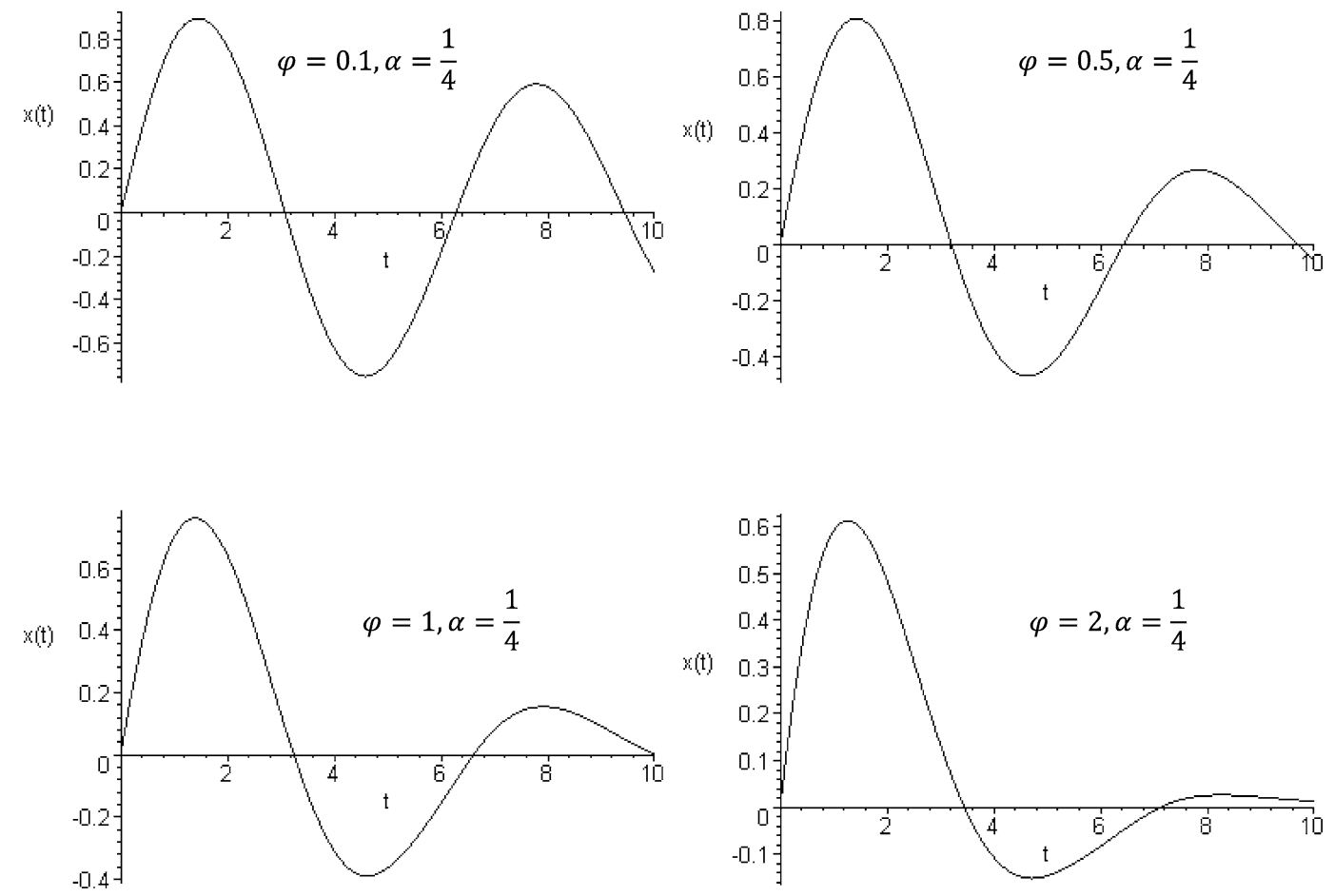

Fig. 6 Plots of displacement $x$ versus time $t$ for $\alpha=\frac{1}{2}$ and different value of $\varphi$ 
(a1)

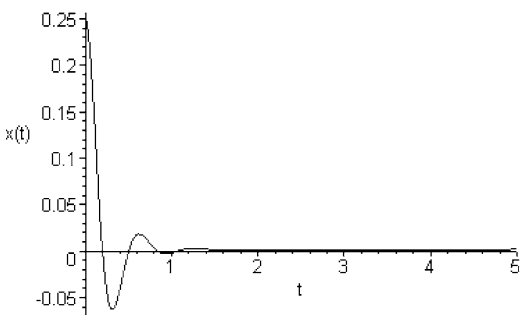

(d1)

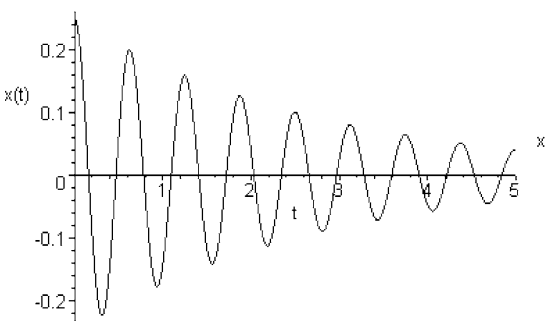

(b1)

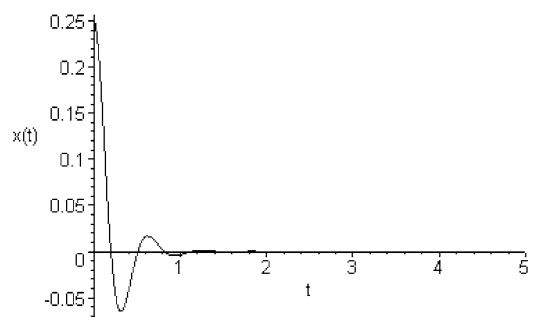

(e1)

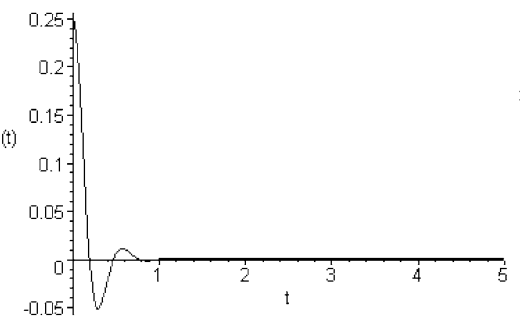

(c1)

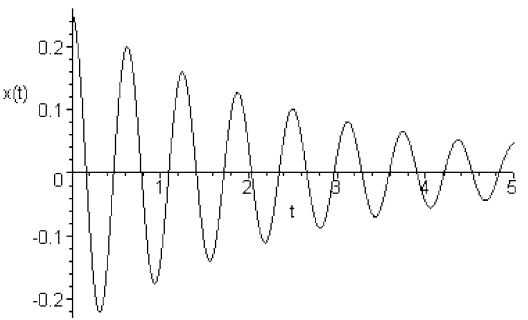

(11)

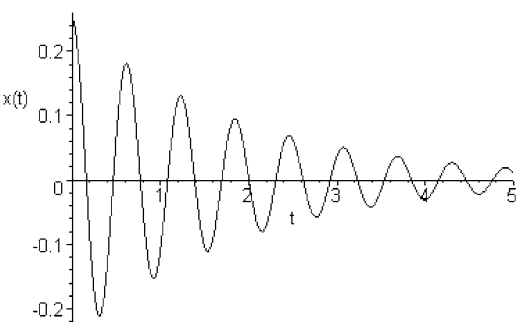

Fig. 7 (a1), (b1), (c1), (d1), (e1) and (f1) solution curves for problems (a), (b), (c), (d), (e) and (f) in Table 4, respectively

Table 3 Approximate solutions for Rayleigh differential equation (23), when $\varphi=0.1$, obtained using multi-step DTM and RK4 method

\begin{tabular}{|c|c|c|c|c|}
\hline \multirow[t]{2}{*}{$t$} & \multicolumn{2}{|l|}{ Multi-step DTM } & \multicolumn{2}{|l|}{ RK4 } \\
\hline & $x(t)_{\alpha=1}$ & $d x / d t_{\alpha=1}$ & $x(t)_{\alpha=1}$ & $d x / d t_{\alpha=1}$ \\
\hline 0 & 1 & 0 & 1 & 0 \\
\hline 1 & 0.5538341229 & -0.8058457634 & 0.55384016928 & -0.80583630162 \\
\hline 2 & -0.3487458062 & -0.8461489739 & -0.34873346751 & -0.84614942123 \\
\hline 3 & -0.8757300446 & -0.1273330986 & -0.87572902070 & -0.12735434293 \\
\hline 4 & -0.5875864331 & 0.6429247994 & -0.58760876750 & 0.64290438106 \\
\hline 5 & 0.1954146215 & 0.7892767357 & 0.19538390707 & 0.78928372686 \\
\hline 6 & 0.7447805008 & 0.2231761103 & 0.74477340850 & 0.22321331192 \\
\hline 7 & 0.5919632131 & -0.4911777525 & 0.59199456555 & -0.49114528277 \\
\hline 8 & -0.0685995951 & -0.7156967960 & -0.06855381787 & -0.71570417824 \\
\hline 9 & -0.6148398107 & -0.2894220838 & -0.61482358172 & -0.28946966851 \\
\hline 10 & -0.5730327815 & 0.3550901319 & -0.57306687530 & 0.35504606729 \\
\hline
\end{tabular}

$$
x(t)= \begin{cases}\sum_{k=0}^{N} X_{0}(k) t^{k \alpha}, & t \in\left[t_{0}, t_{1}\right] \\ \sum_{k=0}^{N} X_{1}(k) t^{k \alpha}, & t \in\left[t_{1}, t_{2}\right] \\ \vdots & \\ \sum_{k=0}^{N} X_{M}(k) t^{k \alpha}, & t \in\left[t_{M-1}, t_{M}\right]\end{cases}
$$

where $X_{i}(n)$, for $n=1 \ldots M$, satisfy the following recurrence relations,

$$
\begin{aligned}
& \frac{\Gamma\left(\frac{k+q+r q}{q}\right)}{\Gamma\left(\frac{k+q}{q}\right)} X_{i}(k+r q) \\
& \quad+2 \mu w_{n}^{2-\frac{p}{q}} \frac{\Gamma\left(\frac{k+q+p}{q}\right)}{\Gamma\left(\frac{k+q}{q}\right)} X_{i}(k+p)+w_{n}^{2} X_{i}(k) \\
& \quad=\frac{f_{0} \sum_{i=0}^{\infty}(-1)^{i} w_{e}^{2 i+1} \delta(k-(2 i+1) q)}{(2 i+1) !}
\end{aligned}
$$

$X_{0}(0)=\frac{1}{4}, \quad X_{0}(q)=0$, 
Table 4 Numerical parameters of the examples [37]

\begin{tabular}{lllllll}
\hline Parameters & (a) & (b) & (c) & (d) & (e) & (f) \\
\hline$m$ & 1 & 1 & 1 & 1 & 1 & 1 \\
$\alpha$ & $\frac{1}{2}$ & $\frac{1}{2}$ & $\frac{1}{2}$ & $\frac{1}{2}$ & $\frac{1}{5}$ & $\frac{1}{5}$ \\
$w_{n}$ & 10 & 10 & 10 & 10 & 10 & 10 \\
$\mu$ & 0.5 & 0.5 & 0.05 & 0.05 & 0.05 & 0.05 \\
$f_{0}$ & 1 & 1 & 1 & 1 & 1 & 1 \\
$w_{e}$ & $4 \pi$ & - & $4 \pi$ & - & $4 \pi$ & $4 \pi$ \\
$x(0)$ & $\frac{1}{4}$ & $\frac{1}{4}$ & 0 & $\frac{1}{4}$ & $\frac{1}{4}$ & $\frac{1}{4}$ \\
$x^{\prime}(0)$ & 0 & 0 & 0 & 0 & 0 \\
\hline
\end{tabular}

Table 5 Approximate solutions for vibration differential equation (27), when $c=1, m=1, k=100, f=1, w_{\theta}=0$, obtained using multi-step DTM and RK4 method

\begin{tabular}{|c|c|c|c|c|}
\hline \multirow[t]{2}{*}{$t$} & \multicolumn{2}{|c|}{ Multi-step DTM } & \multicolumn{2}{|l|}{ RK4 } \\
\hline & $\overline{x(t)_{\alpha=1}}$ & $d x / d t_{\alpha=1}$ & $\overline{x(t)_{\alpha=1}}$ & $d x / d t_{\alpha=1}$ \\
\hline 0.0 & $2.500 \mathrm{e}-01$ & $0.000 \mathrm{e}+00$ & $2.500 \mathrm{e}-01$ & $0.000 \mathrm{e}+00$ \\
\hline 0.5 & $4.470 \mathrm{e}-02$ & $1.873 \mathrm{e}+00$ & $4.470 \mathrm{e}-02$ & $1.873 \mathrm{e}+00$ \\
\hline 1.0 & $-1.323 \mathrm{e}-01$ & $8.100 \mathrm{e}-01$ & $-1.323 \mathrm{e}-01$ & $8.099 \mathrm{e}-01$ \\
\hline 1.5 & $-8.433 \mathrm{e}-02$ & $-7.856 \mathrm{e}-01$ & $-8.433 \mathrm{e}-02$ & $-7.856 e-01$ \\
\hline 2.0 & $4.377 \mathrm{e}-02$ & $-8.311 \mathrm{e}-01$ & $4.378 \mathrm{e}-02$ & $-8.310 \mathrm{e}-01$ \\
\hline 2.5 & $7.008 \mathrm{e}-02$ & $1.170 \mathrm{e}-01$ & $7.008 \mathrm{e}-02$ & $1.171 \mathrm{e}-01$ \\
\hline 3.0 & $3.769 \mathrm{e}-03$ & $5.547 \mathrm{e}-01$ & $3.756 \mathrm{e}-03$ & $5.547 \mathrm{e}-01$ \\
\hline 3.5 & $-4.088 \mathrm{e}-02$ & $1.690 \mathrm{e}-01$ & $-4.088 \mathrm{e}-02$ & $1.689 \mathrm{e}-01$ \\
\hline 4.0 & $-1.997 \mathrm{e}-02$ & $-2.633 \mathrm{e}-01$ & $-1.996 \mathrm{e}-02$ & $-2.634 \mathrm{e}-01$ \\
\hline 4.5 & $1.615 \mathrm{e}-02$ & $-2.165 \mathrm{e}-01$ & $1.616 \mathrm{e}-02$ & $-2.163 e-01$ \\
\hline 5.0 & $1.910 \mathrm{e}-02$ & $6.606 e-02$ & $1.910 \mathrm{e}-02$ & $6.620 \mathrm{e}-02$ \\
\hline
\end{tabular}

$X_{i+1}(0)=X_{i}\left(t^{*}\right), \quad X_{i+1}(q)=X_{i}\left(t^{*}\right)$

$t \in\left[t_{i}, t_{i+1}\right], \quad t^{*}=t_{i}, \quad i=0, \ldots, M-1$

The results in Fig. 7 compatible with those obtained in [37] using the Adomian decomposition method.

For the solution of Eq. (28), the parameter values of Table 4 were used. Table 5 shows the approximate solutions for Eq. (27) obtained for different values of a using MsDTM with the Runge Kutta Method. From the numerical results in Table 5, it is clear that the approximate solutions are in high agreement with the RKM solutions, when $\alpha=1$.

\section{Conclusions}

In this work, we carefully applied the multi-step DTM, a reliable modification of the DTM that improves the convergence of the series solution. The method provides immediate and visible symbolic terms of analytic solutions, as well as numerical solutions for wide classes of linear and nonlinear fractional differential equations. The validity of the proposed method has been successful by applying it for Duffing, The Vander Pol, Rayleigh and vibration equations. The method was used in a direct way without using linearization, perturbation or restrictive assumptions. It provides the solutions in terms of convergent series with easily computable components and the results have shown remarkable performance. Therefore, the proposed method is very efficient and accurate method that can be used to provide analytical solutions for nonlinear fractional-order differential equations. 
Open Access This article is distributed under the terms of the Creative Commons Attribution License which permits any use, distribution, and reproduction in any medium, provided the original author(s) and the source are credited.

\section{References}

1. [Lord Rayleigh] Strutt JW (1945) Theory of sound, 1 (reprint). Dover, New York

2. Mickens RE (1981) A uniformly valid asymptotic solution for $\frac{d^{2} y}{d t^{2}}+y=a+\varepsilon y^{2}$. J Sound Vib 76:150-152

3. Van Woerkom PT (1982) Comments on "A uniformly valid asymptotic solution for $\frac{d^{2} y}{d t^{2}}+y=a+\varepsilon y^{2}$ ". J Sound Vib 80:157-158

4. Kelly SG (1982) Comments on "A uniformly valid asymptotic solution for $\frac{d^{2} y}{d t^{2}}+y=a+\varepsilon y^{2}$ ". J Sound Vib 80:155-156

5. Atadan AS, Huseyin K (1982) A note on "A uniformly valid asymptotic solution for $\frac{d^{2} y}{d t^{2}}+y=a+\varepsilon y^{2}$ ". J Sound Vib 85:129-131

6. Zhou JK (1986) Differential transformation and its applications for electrical circuits. Huazhong University Press, Wuhan, China (in Chinese)

7. Ayaz F (2004) Solutions of the system of differential equations by differential transform method. Appl Math Comput 147:547-567

8. Ayaz F (2004) Application of differential transform method to differential-algebraic equations. Appl Math Comput 152:649-657

9. Arikoglu A, Ozkol I (2005) Solution of boundary value problems for integro-differential equations by using differential transform method. Appl Math Comput 168:1145-1158

10. Bildik N, Konuralp A, Bek F, Kucukarslan S (2006) Solution of different type of the partial differential equation by differential transform method and Adomian's decomposition method. Appl Math Comput 127:551-567

11. Arikoglu A, Ozkol I (2006) Solution of difference equations by using differential transform method. Appl Math Comput 173(1):126-136

12. Arikoglu A, Ozkol I (2006) Solution of differential difference equations by using differential transform method. Appl Math Comput 181(1):153-162

13. Liu H, Song Y (2007) Differential transform method applied to high index differential-algebraic equations. Appl Math Comput 184(2):748-753

14. Momani S, Noor M (2007) Numerical comparison of methods for solving a special fourth-order boundary value problem. Appl Math Lett 191(1):218-224

15. Hassan IH (2008) Comparison differential transformation technique with Adomian decomposition method for linear and nonlinear initial value problems. Chaos Solitons Fractals 36(1):53-65

16. Hassan IH (2008) Application to differential transformation method for solving systems of differential equations. Appl Math Model 32(12):2552-2559

17. El-Shahed M (2008) Application of differential transform method to non-linear oscillatory systems. Commun Nonlinear Sci Numer Simul 13(8):1714-1720
18. Odibat Z (2008) Differential transform method for solving Volterra integral equation with separable kernels. Math Comput Model 48(7-8):144-149

19. Momani S, Odibat Z, Erturk V (2007) Generalized differential transform method for solving a space- and time-fractional diffusion-wave equation. Phys Lett A 370(5-6):379-387

20. Erturk V, Momani S, Odibat Z (2008) Application of generalized differential transform method to multi-order fractional differential equations. Commun Nonlinear Sci Numer Simul 13(8):1642-1654

21. Odibat Z, Momani S (2008) Generalized differential transform method for linear partial differential equations of fractional order. Appl Math Lett 21(2):194-199

22. Momani S, Odibat Z (2008) A novel method for nonlinear fractional partial differential equations: combination of DTM and generalized Taylor's formula. J Comput Appl Math 220(1-2):85-95

23. Odibat Z, Momani S, Erturk V (2008) Generalized differential transform method: application to differential equations of fractional order. Appl Math Comput 197(2):467-477

24. Kuo B, Lo C (2009) Application of the differential transformation method to the solution of a damped system with high nonlinearity. Nonlinear Anal TMA 70(4):1732-1737

25. Al-Sawalha M, Noorani M (2009) Application of the differential transformation method for the solution of the hyperchaotic Rössler system. Commun Nonlinear Sci Numer Simul 14(4):1509-1514

26. Chen S, Chen C (2009) Application of the differential transformation method to the free vibrations of strongly non-linear oscillators. Nonlinear Anal, Real World Appl 10(2):881-888

27. Merdan M, Gökdoğan A (2011) Solution of nonlinear oscillators with fractional nonlinearities by using the modified differential transformation method. Math Comput Appl 16(3):761-772

28. Gökdogan A, Merdan M (2010) A numeric-analytic method for approximating the Holling Tanner model. Stud Nonlin Sci 1(3):77-81

29. Abdel-Halim Hassan IH (2002) On solving some eigenvalue problems by using a differential transformation. Appl Math Comput 127:1-22

30. Odibat ZM, Bertelle C, Aziz-Alaoui MA, Duchamp GHE (2010) A multi-step differential transform method and application to non-chaotic or chaotic systems. Comput Math Appl 59:1462-1472

31. Gökdoğan A, Merdan M, Yıldırım A (2012) Adaptive multi-step differential transformation method to solving nonlinear equations. Math Comput Model 55(3-4):761-769

32. Caputo M (1967) Linear models of dissipation whose Q is almost frequency independent. Part II. J R Aust Soc 13:529-539

33. Momani S, Odibat Z (2006) Analytical approach to linear fractional partial differential equations arising in fluid mechanics. Phys Lett A 355:271-279 
34. Arikoglu A, Ozkol I (2007) Solution of fractional differential equations by using differential transform method. Chaos Solitons Fractals 34(5):1473-1481

35. Oturanç G, Kurnaz A, Keskin Y (2008) A new analytical approximate method for the solution of fractional differential equations. Int J Comput Math 85(1):131-142
36. Cenesiz Y, Keskin Y, Kurnaz A (2010) The solution of the Bagley-Torvik equation with the generalized Taylor collocation method. J Franklin Inst 347:452-466

37. Palfalvi A (2010) Efficient solution of a vibration equation involving fractional derivatives. Int J Non-Linear Mech 45:169-175 